\title{
Malaria
}

\section{Mosquitoes get a leg up on insecticides}

\section{Flaminia Catteruccia}

\author{
A chemosensory protein enriched in the legs of malaria- \\ carrying mosquitoes gives them resistance to insecticides \\ used to treat bed nets. This discovery points to the challenges \\ of tackling malaria. See p.376
}

The wide distribution of insecticide-treated mosquito nets across malaria-endemic regions has drastically reduced the incidence of the disease over the past 20 years, and so has saved millions of lives ${ }^{1}$. However, malaria-carrying mosquitoes have now developed strong resistance to the pyrethroid chemicals used in these long-lasting insecticidal nets (LLINs) ${ }^{2}$. An understanding of the mechanisms underlying resistance should reveal ways to make mosquitoes susceptible to insecticides once more. On page 376 , Ingham et al. ${ }^{3}$ demonstrate an unexpected way in which mosquitoes in Africa neutralize pyrethroids: they use a class of small proteins normally involved in chemical communication.

Malaria parasites are transmitted to humans by female mosquitoes of the genus Anopheles, with Anopheles gambiae being a major carrier of the disease. The first identified mechanism of pyrethroid resistance in wild Anopheles populations was a phenomenon called knockdown resistance, which involves mutations in a voltage-gated sodium channel protein that reduce neuronal sensitivity to the insecticide ${ }^{4}$. Other mechanisms have also been identified, including enhanced metabolic activity of detoxifying enzymes such as cytochrome P450s (CYPs), which bind to and promote the breakdown of insecticides $^{5}$ (Fig. 1).

The emergence of strong pyrethroid resistance in West African populations of A. gambiae ${ }^{6}$ prompted Ingham et al. to search for more mediators of resistance. The authors analysed the gene-expression profiles of insecticide-resistant $A$.gambiae populations from Burkina Faso and Côte d'Ivoire. To the authors' surprise, they discovered higher-than-normal expression of genes that encode a family of chemosensory proteins, called sensory appendage proteins (SAPs).
SAPs, like all chemosensory proteins, are found only in insects. They are small, soluble proteins that typically transmit chemical signals by transporting small hydrophobic molecules between cells. Ingham and colleagues found that reducing the levels of one of these proteins, SAP2, in pyrethroid-resistant $A$.gambiae significantly restored the mosquitoes' susceptibility to pyrethroids. Conversely, overexpressing SAP2 in an otherwise susceptible $A$. gambiae colony boosted the mosquitoes' resistance levels.

How does a chemosensory protein interfere with insecticide activity? Ingham et al. show that SAP2 binds to pyrethroids with high specificity, and that its expression is enhanced in the legs of mosquitoes. These data suggest that SAPs sequester pyrethroids that penetrate the mosquito's hard exterior when it lands on a bed net, perhaps preventing the insecticide from exerting its toxic effect on the nervous system by promoting its breakdown.

Finally, Ingham et al. analysed the genomes of West African Anopheles populations collected over time, making use of an existing database as well as sequences that they had gathered. They found that a 'selective sweep' had occurred near the genomic region that encodes SAP2 - a phenomenon in which one particular version of a genomic region becomes more prevalent in a population as a result of natural selection. The authors showed that the sweep had occurred over the time in which pyrethroid resistance sharply increased, maybe owing to the beneficial effects of one version of this genomic region on survival. Taken together, Ingham and colleagues' data unveil chemosensory proteins

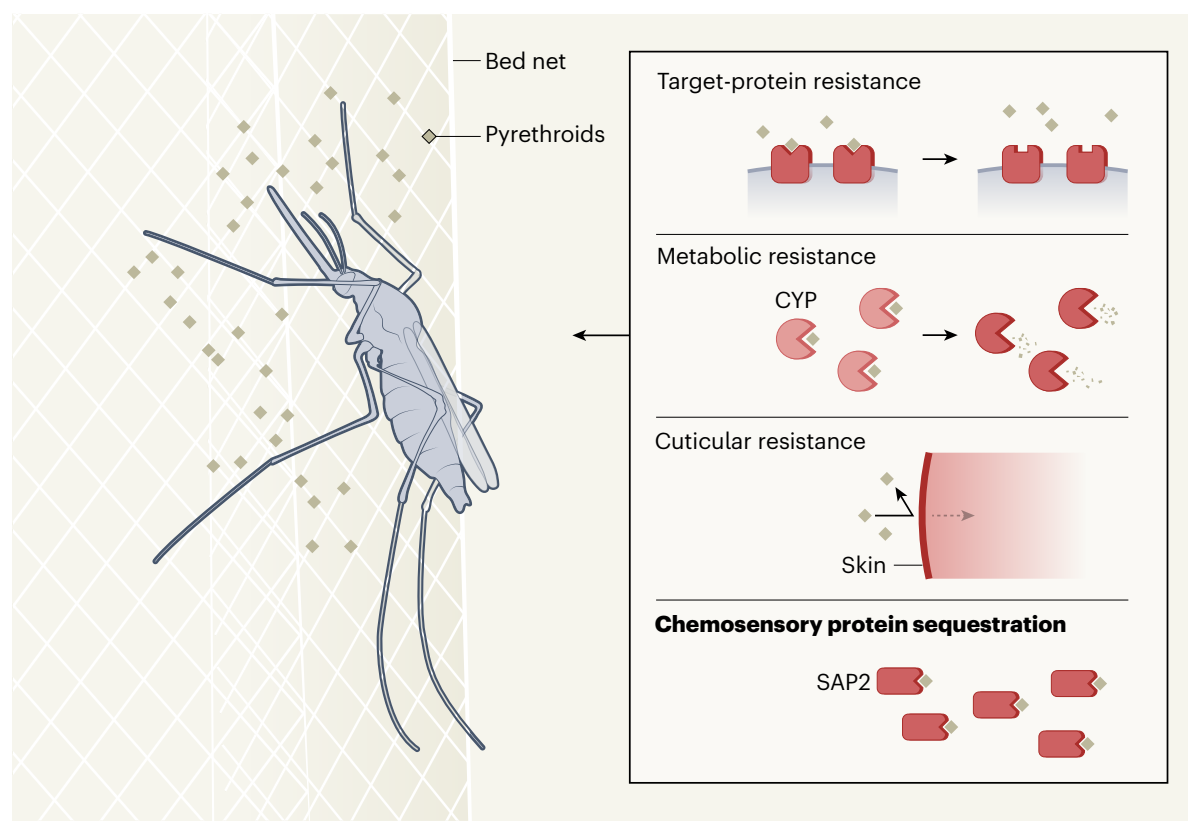

Figure 1 | Multiple lines of resistance. Malaria-carrying mosquitoes in West Africa have developed several mechanisms of resistance to insecticides called pyrethroids, which are used to treat bed nets ${ }^{2}$. Mutations in a voltage-gated sodium channel protein to which pyrethroids bind reduce the chemicals' ability to recognize their target. Enhanced activity of metabolic enzymes such as the cytochrome P450s (CYPs) can break down the pyrethroids before they kill the insect. Thickening of the mosquito's outer cuticular layer can slow insecticide penetration into the skin, thereby reducing the chemicals' concentration. Ingham et al. ${ }^{3}$ now show that, in the insect's legs, the chemosensory protein SAP2 binds to pyrethroids, sequestering them and so preventing them from functioning. 
as a key component of insecticide resistance in Anopheles mosquitoes.

Chemosensory proteins represent a previously unknown class of resistance-causing factor, and so Ingham and co-workers' finding points to a fresh opportunity to restore full susceptibility to pyrethroids in West African Anopheles populations. Resistance caused by CYP enzymes has been mitigated by adding compounds that interfere with CYPs to bed nets ${ }^{7}$; similarly, compounds that inhibit binding between SAP 2 and insecticides could be generated and incorporated into next-generation LLINs. Moreover, the genomic region associated with SAP2 resistance can now be used as a molecular marker for tracking the spread of this resistance mechanism. In the future, it will be crucial to determine whether and how chemosensory proteins interact both functionally and spatially with other mechanisms of resistance, to inform the optimal design of resistance-management strategies.

Although Ingham and co-workers' study provides hope of reversing mosquitoes' resistance to insecticides, it also highlights how skilled these insects are at escaping unwanted attention. Clearly, our understanding of insecticide resistance is far from complete, and we should expect other studies in different locations to identify yet more such mechanisms, acting at local or continental levels. Anopheles species have been populating Africa for more than 100 million years considerably longer than humans and our ancestors ${ }^{8}$. Such an enduring connection with their natural habitat is a testament to the challenge that we face when targeting these insects.

The next generation of LLINs and indoor residual sprays (another method of delivering insecticides) is currently being deployed in Africa ${ }^{9}$. Simultaneously, new insecticide-based methods such as insect-attractive targeted sugar baits are being tested ${ }^{10}$. But, like previous interventions, these tools will probably undergo cycles of impactful but relatively short-lived success, followed by decreased effectiveness owing to the emergence of resistance. Beyond insecticides, other mosquito-control strategies will probably encounter similar resistance issues. These include methods that rely on mosquito killing, such as mosquitocidal drugs ${ }^{11}$, and genetic systems designed to suppress Anopheles populations (reviewed in ref.12).

It is possible that the combined use of multiple strategies will break mosquito endurance and lead to population collapse. However, work from my group recently showed that strong selective pressures imposed on Anopheles females could actually favour malaria transmission, for instance by triggering acceleration of parasite growth rates ${ }^{13}$. To avoid this issue, mosquito-targeting interventions could be integrated with approaches that block parasite development in the insects without causing them harm, thus reducing selective pressures. Furthermore, mathematical models suggest that our chances of achieving sustainable malaria control could be improved by incorporating antimalarials into LLINs or indoor residual sprays, to kill parasites and prevent their transmission even when mosquitoes become resistant to insecticides ${ }^{14}$. Similar results could be obtained by delivering antiparasitic agents through biological or genetic means ${ }^{12}$.

Whatever the eventual solution, the road to malaria elimination remains long. Mosquitoes are sending clear signals that they will fight for their survival.

Flaminia Catteruccia is in the Department of Immunology and Infectious Diseases, Harvard
T.H. Chan School of Public Health, Boston,

Massachusetts 02115, USA.

e-mail: fcatter@hsph.harvard.edu

Bhatt, S. et al. Nature 526, 207-211 (2015).

2. Ranson, H. \& Lissenden, N. Trends Parasitol. 32, 187-196 (2016).

3. Ingham, V. A. et al. Nature 577, 376-380 (2020).

4. Martinez-Torres, D. et al. Insect Mol. Biol. 7, 179-184 (1998).

5. Stevenson, B. J. et al. Insect Biochem. Mol. Biol. 41, 492-502 (2011).

6. Churcher, T. S. Lissenden, N., Griffin, J. T., Worrall, E. \& Ranson, H. elife 5, e1609o (2016).

7. Protopopoff, N. et al. Lancet 391, 1577-1588 (2018).

8. Neafsey, D. E. et al. Science 347, 1258522 (2015).

9. N'Guessan, R., Odjo, A., Ngufor, C., Malone, D. \& Rowland, M. PLOS ONE 11, e0165925 (2016).

10. Qualls, W. A. et al. Malar. J. 14, 301 (2015).

11. Kobylinski, K. C. et al. Acta Trop. 116, 119-126 (2010).

12. Shaw, W. R. \& Catteruccia, F. Nature Microbiol. 4, 20-34 (2019).

13. Werling, K. et al. Cell 177, 315-325 (2019).

14. Paton, D. G. et al. Nature 567, 239-243 (2019).

\title{
Nanotechnology
}

\section{Evolution of circuits for machine learning}

\author{
Cyrus F. Hirjibehedin
}

The fundamental machine-learning task of classification
can be difficult to achieve directly in ordinary computing
hardware. Unconventional silicon-based electrical circuits
can be evolved to accomplish this task. See p.341

Artificial intelligence (AI) has allowed computers to solve problems that were previously thought to be beyond their capabilities, from defeating the best human opponents in complex games ${ }^{1}$ to automating the identification of diseases ${ }^{2}$. There is therefore great interest in developing specialized circuits that can complete AI calculations faster and with lower energy consumption than can current devices. On page 341 , Chen et al. ${ }^{3}$ demonstrate an unconventional electrical circuit in silicon that can be evolved in situ to carry out basic machine-learning operations.

Although computers excel at performing calculations that have well-defined answers, they have not been good at making guesses. For example, if you are thinking about selling your car, a computer is ideally suited for calculating the average price that similar cars have sold for, to help you determine your selling price. But by analysing the enormous digital data sets that are currently available, AI techniques such as machine learning can now teach computers to make sensible predictions. One of the most basic operations that machine-learning algorithms can carry out when provided with a large set of inputs (such as the age of a car and how many kilometres it has been driven) is classification into one of a set of categories, such as whether the car is in poor, fair or good condition and therefore whether you can expect to get the price you want for it.

Using the structure of the human brain as inspiration, scientists and engineers have made substantial progress in developing specialized hardware to greatly reduce the amount of time and energy needed to perform tasks such as classification ${ }^{4}$. There are also many unconventional device concepts for machine learning that are still in the early stages of development but that could offer radical new capabilities. For example, researchers are exploring whether superconductor-based electrical circuits that work at only a few degrees above absolute zero, and that operate at gigahertz frequencies with high energy efficiency, could enable machine-learning applications that are currently infeasible using conventional approaches ${ }^{5}$.

Chen and co-workers' circuit is also inspired by the brain, and represents a major departure from typical electrical circuits. Normally, electrical current flows through circuits like water flowing in a river. If the river becomes so shallow that it is reduced to a set of small 